\title{
Algebraic Structure and Poisson Integral Method of Snake-Like Robot Systems
}

\author{
Fu Jing-Li ${ }^{1 *}$, Xiang Chun ${ }^{1}$ and Meng Lei ${ }^{2}$ \\ ${ }^{1}$ College of Mechanical and Automotive Engineering, Zhejiang University of Water Resources and Electric Power, Hangzhou, \\ China, ${ }^{2}$ Institute of Mathematical Physics, Zhejiang Sci-Tech University, Hangzhou, China
}

The algebraic structure and Poisson's integral of snake-like robot systems are studied. The generalized momentum, Hamiltonian function, generalized Hamilton canonical equations, and their contravariant algebraic forms are obtained for snake-like robot systems. The Lie-admissible algebra structures of the snake-like robot systems are proved and partial Poisson integral methods are applied to the snake-like robot systems. The first integral methods of the snake-like robot systems are given. An example is given to illustrate the results.

Keywords: snake-like robot, algebraic structure, poisson integral, lagrange equation, hamiltonian function

OPEN ACCESS

Edited by:

Yang-Hui He,

City University of London,

United Kingdom

Reviewed by:

Alberto Molgado,

Autonomous University of San Luis

Potosí, Mexico

Q. H. Liu,

Hunan University, China

*Correspondence:

Fu Jing-Li

sqfujingli@163.com

Specialty section:

This article was submitted to Mathematical and Statistical Physics,

a section of the journal

Frontiers in Physics

Received: 18 December 2020 Accepted: 12 April 2021

Published: 10 June 2021

Citation:

Jing-Li F, Chun X and Lei M (2021) Algebraic Structure and Poisson Integral Method of Snake-Like Robot

Systems. Front. Phys. 9:643016.

doi: 10.3389/fphy.2021.643016

\section{INTRODUCTION}

The snake-like robot, which is based on the biological characteristics of snakes, constitutes an important branch of bionic robots [1]. Hirose developed the first snake robot in 1972 [2]. The snake robot is significantly different from a tracked robot, wheeled robot, and legged robot, being a mobile robot with high redundancy. Because of the multi-joint flexible structure design, a snake robot has the advantage of multi-gait motion and the ability to adapt to a complex unknown environment, and can be widely used in disaster rescue, underwater surveys, industrial testing, and other special environments that traditional robots or humans cannot enter; as a result, increasing attention is being paid to snake robots [3-6].

In 1946, Gray divided movement gaits into serpentine movement, rectilinear movement, concertina movement, and sidewinding movement in the study of the biological nature of snakes [7]. According to this study, there are two starting points to study the motion of the snake-like robot: One is to observe the movement rule of biological snakes from the perspective of bionics, and then apply the rule to the snake-like robot to verify its effectiveness and controllability; on the other hand, the physical models are established according to the actual physical systems, and based on the physical model a control law is proposed to make snake-like robots move in a serpentine motion. For example, Tang et al. [8] studied the control methods of snake-like robots in different environments. Hirose established a serpentine gait kinematics model with linkage structures based on the observation of biological snake movement processes and bone anatomy [2], Lilijeback et al. analyzed the position relationship between a snake robot and obstacles, proposed an obstacle assistant movement gait in planar motion, and built the kinematics and dynamics model for the snake robot $[9,10]$. At present, the serpentine motion of many snake-like robots are realized on the passive wheel, while the passive wheel provides a non-holonomic constraint for the system of snake-like robots in dynamics, so it is necessary to analyze and discuss the constraint systems of snake-like robots. Ostrowski and Burdick [11] and Guo et al. [12] developed the kinematic model considering the constraint systems of snake-like robots. 


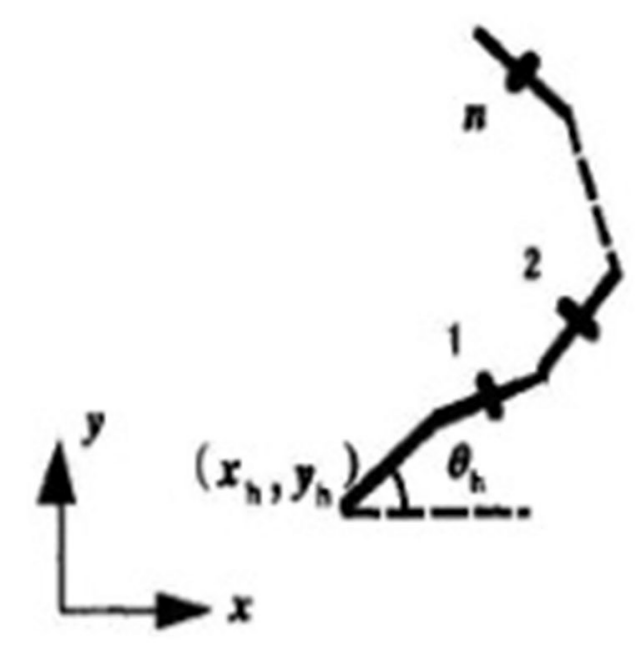

FIGURE 1 | Mechanical structure of snake-like robot.

It is well-known that algebraic structure and Poisson's theory for constrained mechanical systems have been developed to seek invariants of mechanical and physical systems [13, 14]. Mei and Shi [15] have extended this method to non-holonomic constrained mechanical systems. Fu et al. have studied the algebraic structure and Poisson's theory of the relativistic Birkhoffian system, rotational relativistic dynamical system, mechanico-electrical coupling system, and $f(R)$ cosmology system [16-18]. In this paper, we make an effort in this direction and demonstrate the applications of the algebraic structure and Poisson's theory of dynamical systems to snake-like robot system. This paper gives the algebraic structure and the first integral methods of snake-like robots. Firstly, the kinematics of the snake-like robot is analyzed, and then the generalized Lagrange equations and generalized Hamilton canonical equations of the snake-like robot systems are given. Secondly, contravariant algebraic forms of snake-like robot systems are obtained. Thirdly the Lie-admissible algebraic structure of the snake-like robot is researched. Fourthly, the Poisson integral methods are applied to the snake-like robot systems. Finally, an example is studied for a snake-like robot.

\section{LAGRANGIAN OF SNAKE-LIKE ROBOT SYSTEMS}

In order to facilitate the analysis, the snake-like robot systems are simplified as a link mechanism, each link rod represents a joint of the snake-like robot (mechanical structure of snake-like robot systems are depicted in Figure 1). Then, according to Figure 1, we can get the analysis as follows: let $\left(x_{i}, y_{i}\right)$ denote the center of gravity of each joint (each coordinate is set in the middle of each link), $\varphi_{i}$ is the angle between the link and the horizontal direction, $\left(x_{h}, y_{h}\right)$ and the $\theta_{h}$ denote the position of the snakelike robot and the absolute angle of snake-head, respectively, and

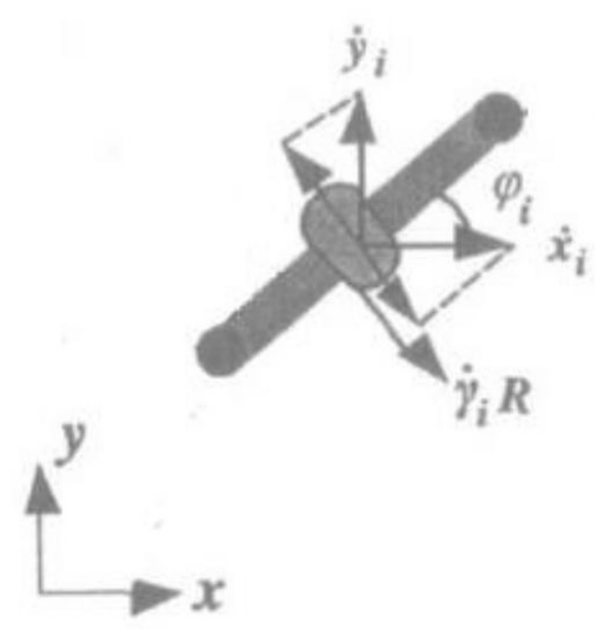

FIGURE 2 | A simplified model of one unit of the snake-like robot.

$m_{h}$ denotes the weight of the head of the robot. Length $2 l$ and weight $m$ are the same for each link.

We choose $q_{i}=\varphi_{i}, q_{n+1}=x_{h}, q_{n+2}=y_{h}, q_{n+3}=\theta_{h}$, $(i=1,2, \cdots, n)$ as the generalized coordinates.

From Figure 1, the relationship between the center of gravity of each joint $\left(x_{i}, y_{i}\right)$ and the position of the snake head $\left(x_{h}, y_{h}\right)$ can be given by

$$
\begin{gathered}
x_{i}=q_{n+1}+2 l \cos \left(q_{n+3}\right)+2 l \sum_{k=1}^{i-1} \cos \left(q_{k}\right)+l \cos \left(q_{i}\right), \\
y_{i}=q_{n+2}+2 l \sin \left(q_{n+3}\right)+2 l \sum_{k=1}^{i-1} \sin \left(q_{k}\right)+l \sin \left(q_{i}\right),
\end{gathered}
$$

so, the generalized velocities of snake-like systems can be given by

$\dot{x}_{i}=7 \dot{q}_{n+1}-2 l \sin \left(q_{n+3}\right) \dot{q}_{n+3}-2 l \sum_{k=1}^{i-1} \sin \left(q_{k}\right) \dot{q}_{k}-l \sin \left(q_{i}\right) \dot{q}_{i}$,
$\dot{y}_{i}=\dot{q}_{n+2}+2 l \cos \left(q_{n+3}\right) \dot{q}_{n+3}+2 l \sum_{k=1}^{i-1} \cos \left(q_{k}\right) \dot{q}_{k}+l \cos \left(q_{i}\right) \dot{q}_{i}$.

We give a simplified model of one unit of the snake-like robot system as shown in Figure 2.

The constraint equations of the snake-like robot system are given by

$$
\dot{x}_{i} \sin \left(q_{i}\right)-\dot{y}_{i} \cos \left(q_{i}\right)=\dot{q}_{n+3+i} R
$$

where $q_{n+3+\mathrm{i}}=\gamma_{i}$ denotes the rotating angle of each unit's sideslip, $R$ denotes the turning radius of the unit;

submitting constraints (2) into (3), we can derive

$f_{\beta}(q, \dot{q})=\dot{q}_{n+1} \sin \left(q_{i}\right)-\dot{q}_{n+2} \cos \left(q_{i}\right)-2 l \cos \left(q_{n+3}-q_{i}\right) \dot{q}_{n+3}$ 


$$
-2 l \sum_{k=1}^{i-1} \cos \left(q_{i}-q_{k}\right) \dot{q}_{k}-l \dot{q}_{i}-\dot{q}_{n+3+i} R=0 .
$$

The symbols cos and sin are written as $C$ and $S$ respectively, then the kinetic energy of the snake-like robot system is given by

$$
\begin{aligned}
& T=\frac{1}{2}\left[m_{h}\left(\dot{q}_{n+1}^{2}+\dot{q}_{n+2}^{2}\right)+J_{h} \dot{q}_{n+3}^{2}\right]+\frac{1}{2} m \sum_{i=1}^{n}\left[\dot{q}_{n+1}^{2}+\dot{q}_{n+2}^{2}\right. \\
& +4 l^{2} \dot{q}_{n+3}^{2}+4 l^{2}\left[\left(\sum_{k=1}^{i-1} S\left(q_{k}\right) \dot{q}_{k}\right)^{2}+\left(\sum_{k=1}^{i-1} C\left(q_{k}\right) \dot{q}_{k}\right)^{2}\right] \\
& +l^{2} \dot{q}_{2}^{2}+4 l \dot{q}_{n+3}\left(\dot{q}_{n+3} C\left(q_{n+3}\right)-\dot{q}_{n+1} S\left(q_{n+3}\right)\right) \\
& +4 \dot{q}_{n+2} \sum_{k=1}^{i-1} C\left(q_{k}\right) \dot{q}_{k}-4 \dot{q}_{n+1} \sum_{k=1}^{i-1} S\left(q_{k}\right) \dot{q}_{k}+2 \dot{q}_{n+2} C\left(q_{i}\right) \dot{q}_{i} \\
& -2 l \dot{q}_{n+1} S\left(q_{i}\right) \dot{q}_{i}+8 l^{2} \dot{q}_{n+3} S\left(q_{n+3}\right) \sum_{k=1}^{i-1} S\left(q_{k}\right) \dot{q}_{k}+8 l^{2} \dot{q}_{n+3} \\
& C\left(q_{n+3}\right) C \dot{q}_{k}+4 l^{2} \dot{q}_{n+3} \dot{q}_{2} S\left(q_{n+3}\right) S\left(q_{k}\right) \\
& +4 l^{2} \dot{q}_{n+3} \dot{q}_{2} C\left(q_{n+3}\right) C\left(q_{k}\right)+4 l^{2} S\left(q_{i}\right) \dot{q}_{i} \sum_{k=1}^{i-1} S\left(q_{k}\right) \dot{q}_{k} \\
& \left.+4 l^{2} C\left(q_{i}\right) \dot{q}_{i} \sum_{k=1}^{i-1} C\left(q_{k}\right) \dot{q}_{k}\right]+\frac{1}{2} \sum_{i=1}^{n} J_{y} \dot{q}_{i}^{2}+J_{\gamma} \dot{q}_{n+3+i}^{2}, \\
& (i=1, \ldots, n) .
\end{aligned}
$$

The potential energy of the snake-like robot system is assumed to be

$$
U=0 \text {. }
$$

The dissipative functions of the snake-like robot system are assumed to be given by

$$
\begin{aligned}
& D=\frac{1}{2} D_{x y} \sum_{i=1}^{n}\left[\dot{q}_{n+1}^{2}+\dot{q}_{n+2}^{2}+4 l^{2} \dot{q}_{n+3}^{2}\right. \\
& +4 l^{2}\left[\left(\sum_{k=1}^{i-1} S\left(q_{k}\right) \dot{q}_{k}\right)^{2}+\left(\sum_{k=1}^{i-1} C\left(q_{k}\right) \dot{q}_{k}\right)^{2}\right] \\
& +l^{2} \dot{q}_{2}^{2}+4 \dot{q}_{n+3}\left(\dot{q}_{n+3} C\left(q_{n+3}\right)-\dot{q}_{n+1} S\left(q_{n+3}\right)\right) \\
& +4 l \dot{q}_{n+2} \sum_{k=1}^{i-1} C\left(q_{k}\right) \dot{q}_{k}-4 l \dot{q}_{n+1} \sum_{k=1}^{i-1} S\left(q_{k}\right) \dot{q}_{k}+2 l \dot{q}_{n+2} C\left(q_{i}\right) \dot{q}_{i} \\
& -2 l \dot{q}_{n+1} S\left(q_{i}\right) \dot{q}_{i}+8 l^{2} \dot{q}_{n+3} S\left(q_{n+3}\right) \sum_{k=1}^{i-1} S\left(q_{k}\right) \dot{q}_{k}+8 l^{2} \dot{q}_{n+3} \\
& C\left(q_{n+3}\right) \sum_{k=1}^{i-1} C\left(q_{k}\right) \dot{q}_{k}+4 l^{2} \dot{q}_{n+3} \dot{q}_{2} S\left(q_{n+3}\right) S\left(q_{k}\right) \\
& +4 l^{2} \dot{q}_{n+3} \dot{q}_{2} C\left(q_{n+3}\right) C\left(q_{k}\right)+4 l^{2} S\left(q_{i}\right) \dot{q}_{i} \sum_{k=1}^{i-1} S\left(q_{k}\right) \dot{q}_{k}
\end{aligned}
$$

$$
\left.+4 l^{2} C\left(q_{i}\right) \dot{q}_{i} \sum_{k=1}^{i-1} C\left(q_{k}\right) \dot{q}_{k}\right]+\frac{1}{2} \sum_{i=1}^{n} D_{y} \dot{q}_{i}^{2} .
$$

The Routh equation of the snake-like robot system can be given by

$$
\begin{aligned}
\frac{d}{d t} \frac{\partial T}{\partial \dot{q}_{s}}-\frac{\partial T}{\partial q_{s}}= & \mathrm{Q}_{s}+\sum_{\beta=1}^{n} \lambda_{\beta} \frac{\partial f_{\beta}}{\partial \dot{q}_{s}} \\
& (s=1, \cdots, 2 n+3 ; \beta=1, \cdots, n) .
\end{aligned}
$$

After derivation of the constraints of the snake-like robot system (4), we have

$$
\dot{f}_{\beta}(q, \dot{q})=0
$$

From Equation (8) and (9), the Lagrange multiply $\lambda$ can be calculated as

$$
\lambda=\left\{\lambda_{1}, \lambda_{2}, \ldots, \lambda_{n}\right\}
$$

Submitting (10) into (8), we can derive

$$
\frac{d}{d t} \frac{\partial T}{\partial \dot{q}_{s}}-\frac{\partial T}{\partial q_{s}}=Q_{s}+\Lambda_{s},(s=1, \cdots, 2 n+3)
$$

where $\Lambda_{s}=\sum_{\beta=1}^{n} \lambda_{\beta} \frac{\partial f_{\beta}}{\partial \dot{q}_{s}}$.

The motion equation (12) of the complete system corresponding to the snake-like robot system $(4,11)$ can be given as

$$
\frac{d}{d t} \frac{\partial L}{\partial \dot{q}_{s}}-\frac{\partial L}{\partial q_{s}}=Q_{s}{ }^{\prime}+\Lambda_{s}
$$

where $Q_{s}^{\prime}=-\frac{\partial D_{s}}{\partial \dot{q}_{s}}+\tau-\frac{\partial U}{\partial q_{s}}$ are the non-potential force, $\Lambda_{s}=$ $\lambda_{\beta} \frac{\partial f_{\beta}}{\partial \dot{q}_{s}}$ the generalized nonholonomic constraint anti-forces, $L=$ $T-U$ the Lagrangian of the snake-like robot system.

\section{GENERALIZED HAMILTON CANONICAL EQUATIONS OF SNAKE-LIKE ROBOT SYSTEMS}

Assume that the generalized momentums of snake-like robot systems are

$$
\begin{aligned}
& p_{1}=\frac{\partial L}{\partial \dot{q}_{1}}=\frac{1}{2}\left[\left(-4 m(n-1) l S\left(q_{n+3}\right)-2 m l S\left(q_{n+3}+q_{1}\right)\right) \dot{q}_{n+1}\right. \\
& +\left(4 m(n-1) l C\left(q_{n+3}\right)+2 m l C\left(q_{n+3}+q_{1}\right)\right) \dot{q}_{n+2} \\
& +\left(2 m(2+4(n-1)) l^{2} C_{h 1}\right) \dot{q}_{n+3} \\
& \left.+\left(8 m(n-1) l^{2}+2 m l^{2}+2 J_{y}\right) \dot{q}_{1}+\cdots+\left(4 m l^{2} C_{1 n}\right) \dot{q}_{n}\right] \\
& p_{2}=\frac{\partial L}{\partial \dot{q}_{2}}=\frac{1}{2}\left[\left(-4 m(n-2) l S\left(q_{n+3}\right)-2 m l S\left(q_{2}\right)\right) \dot{q}_{n+1}\right. \\
& +\left(4 m(n-2) l C\left(q_{n+3}\right)+2 m l C\left(q_{n+3}+q_{1}\right)\right) \dot{q}_{n+2} \\
& +\left(2 m(2+4(n-2)) l^{2} C_{h 2}\right) \dot{q}_{n+3}
\end{aligned}
$$




$$
\begin{aligned}
& +\left(2 m(2+4(n-2)) l^{2} C_{12}\right) \dot{q}_{1}+\left(8 m(n-2) l^{2}+2 m l^{2}+2 J_{y}\right) \\
& \left.\dot{q}_{2}+\cdots+\left(4 m l^{2} C_{2 n}\right) \dot{q}_{n}\right] \text {, } \\
& p_{n}=\frac{\partial L}{\partial \dot{q}_{n}}=\frac{1}{2}\left[\left(-2 m l s\left(q_{n+3}+\sum_{k=1}^{n} q_{k}\right)\right) \dot{q}_{n+1}\right. \\
& +\left(-2 m l C\left(q_{n+3}+\sum_{k=1}^{n} q_{k}\right)\right) \dot{q}_{n+2} \\
& \left.+\left(4 m l^{2} C_{h 2}\right) \dot{q}_{n+3}+\left(4 m l^{2} C_{1 h}\right) \dot{q}_{1}+\cdots+\left(2 m l^{2}+2 J_{y}\right) \dot{q}_{n}\right], \\
& p_{n+1}=\frac{\partial L}{\partial \dot{q}_{n+1}}=\frac{1}{2}\left[\left(2 m_{h}+2 m n\right) \dot{q}_{n+1}+(0) \dot{q}_{n+2}\right. \\
& +\left(-4 m n l S\left(q_{n+3}\right)\right) \dot{q}_{n+3} \\
& +\left(-4 m(n-1) l S\left(q_{n+3}\right)-2 m l S\left(q_{n+3}+q_{1}\right)\right) \dot{q}_{1}+\cdots+ \\
& \left.\left(-2 m l s\left(q_{n+3}+\sum_{k=1}^{n} q_{k}\right)\right) \dot{q}_{n}\right] \text {, } \\
& p_{n+2}=\frac{\partial L}{\partial \dot{q}_{n+2}}=\frac{1}{2}\left[(0) \dot{q}_{n+1}+(0) \dot{q}_{n+2}+\left(4 m n l C\left(q_{n+3}\right)\right) \dot{q}_{n+3}\right. \\
& +\left(4 m(n-1) l C\left(q_{n+3}\right)+2 m l C\left(q_{n+3}+q_{1}\right)\right) \dot{q}_{1}+\cdots+ \\
& \left.\left(-2 m l C\left(q_{n+3}+\sum_{k=1}^{n} q_{k}\right)\right) \dot{q}_{n}\right] \text {, } \\
& p_{n+3}=\frac{\partial L}{\partial \dot{q}_{n+3}}=\frac{1}{2}\left[\left(-4 m n l S\left(q_{n+3}\right)\right) \dot{q}_{n+1}\right. \\
& +\left(4 m n l C\left(q_{n+3}\right)\right) \dot{q}_{n+2}+\left(2 m_{h}+2 m n\right) \dot{q}_{n+3} \\
& \left.+\left(2 m(2+4(n-1)) l^{2} C_{h 1}\right) \dot{q}_{1}+\cdots+\left(4 m l^{2} C_{h n}\right) \dot{q}_{n}\right] \text {, } \\
& p_{n+4}=\frac{\partial L}{\partial \dot{q}_{n+4}}=J_{r} \dot{q}_{n+4} \text {, } \\
& p_{2 n+3}=\frac{\partial L}{\partial \dot{q}_{2 n+3}}=J_{r} \dot{q}_{2 n+3} .
\end{aligned}
$$

And introduce the Hamiltonian of the snake-like robot system as

$$
\begin{aligned}
H(t, q, \dot{q})= & p_{s} \dot{q}_{s}-L=p_{s} \dot{q}_{s}(t, q, p)-L\left(t, q_{s}, \dot{q}_{s}(t, q, p)\right) \\
& =H(t, q, p)
\end{aligned}
$$

where $p=\left\{p_{1}, p_{2}, \cdots, p_{2 n+3}\right\}$ denotes generalized momentums, equation (16) can be partially regularized as

$$
\begin{aligned}
\dot{q}_{s}= & \frac{\partial H}{\partial p_{s}}, \dot{p}_{s}=-\frac{\partial H}{\partial q_{s}}+Q_{s}{ }^{\prime}+\Lambda_{s} \dot{q}_{s}=\dot{q}_{s}(t, q, p), \\
& (s=1, \cdots, 2 n+3)
\end{aligned}
$$

which is called the generalized Hamilton canonical equation of snake-like robot systems.

\section{CONTRAVARIANT ALGEBRAIC FORMS OF SNAKE-LIKE ROBOT SYSTEMS}

For snake-like robot systems, we can introduce contravariant vectors

$$
a^{\mu}=\left\{\begin{array}{cc}
q^{\mu} & (\mu=1, \cdots, 2 n+3), \\
p_{\mu-n} & (\mu=2 n+4, \cdots, 4 n+6)
\end{array}\right.
$$

then the Hamiltonian of snake-like robot systems will be written in the form

$$
H\left(t, q_{s}, p_{s}\right)=H\left(t, a^{\mu}\right) .
$$

For generalized Hamilton canonical equation (15) of snake-like robot systems, we let

$$
\begin{aligned}
& \left(Q_{s}{ }^{\prime}+\Lambda_{s}\right) \phi_{s}=q_{s}(t, q, p)=\Lambda_{s}^{\prime}=-\Omega_{s k} \frac{\partial H}{\partial p_{k}} \\
& (s, k=1, \cdots, 2 n+3),
\end{aligned}
$$

where

$$
\Omega_{s k}=\left(\begin{array}{cccc}
\Omega_{11} & 0 & \cdots & 0 \\
0 & \Omega_{22} & \cdots & 0 \\
\vdots & \vdots & \ddots & \vdots \\
0 & 0 & \cdots & \Omega_{(2 n+3)(2 n+3)}
\end{array}\right) \text {, }
$$

then Equation (15) can be expressed in the contravariant algebraic form

$$
\dot{a}^{\mu}-S^{\mu \nu} \frac{\partial H}{\partial a^{v}}=0, \quad(\mu, v=1, \cdots, 4 n+6)
$$

where

$$
\begin{aligned}
S^{\mu \nu} & =\omega^{\mu \nu}+T^{\mu \nu} \\
\omega^{\mu \nu} & =\left(\begin{array}{cc}
0_{(2 n+3)(2 n+3)} & I_{(2 n+3)(2 n+3)} \\
-I_{(2 n+3)(2 n+3)} & 0_{(2 n+3)(2 n+3)}
\end{array}\right), \\
T^{\mu \nu} & =\left(\begin{array}{cc}
0_{(2 n+3)(2 n+3)} & 0_{(2 n+3)(2 n+3)} \\
0_{(2 n+3)(2 n+3)} & -\Omega_{k k}
\end{array}\right) .
\end{aligned}
$$

It is obvious that the tensor $S^{\mu \nu}$ is composed of anti-symmetrical tensor $\omega^{\mu \nu}$ and symmetrical tensor $T^{\mu \nu}$.

\section{ALGEBRAIC STRUCTURE OF SNAKE-LIKE ROBOT SYSTEMS}

Firstly, we study the algebraic structure of snake-like robot systems.

Performing the full derivative of function $A(a)$ along Equation (27), and this derivative is defined as a product:

$$
\dot{A}(a)=\frac{\partial A}{\partial a_{\mu}} S^{\mu \nu} \frac{\partial H}{\partial a^{\nu}} \stackrel{\operatorname{def}}{=} A \circ H, \quad(\mu, v=1, \cdots, 2 n+3)
$$

this product satisfies the right-hand assignment law, left-hand assignment law, and scalar law, so we can derive that the snakelike robot system possesses a compatible algebraic structure.

If the snake-like robot system in Equation (27) possesses the Lie algebraic structure, then Equation (31) satisfies the antisymmetrical property

$$
A \circ B+B \circ A=0
$$


and Jacobi identical equation

$$
[A,[B, C]]+[B,[C, A]]+[C,[A, B]]=0 .
$$

When considering Equations (28)-(30), Equations (32) and (33) lead to a condition with respect to $T^{\mu \nu}$

$$
\begin{aligned}
& T^{\mu \nu}+T^{\nu \mu}=0 \\
& T^{\tau \rho} \frac{\partial T^{\mu \nu}}{\partial a^{\rho}}+T^{\mu \rho} \frac{\partial T^{\nu \tau}}{\partial a^{\rho}}+T^{\nu \rho} \frac{\partial T^{\tau \mu}}{\partial a^{\rho}}=0, \\
& (\mu, \nu, \tau, \rho=1, \cdots, 4 n+6) .
\end{aligned}
$$

Further, we have $\Omega_{k k}=0, Q_{s}=0,(s, k=1, \cdots, 2 n+3)$. Then Equation (27) has no Lie algebraic structure, and we have

Conclusion 1: The snake like-robot systems do not have Lie algebraic structure.

For Equation (31), we define a new product

$$
[A, B] \stackrel{\text { def }}{=} A \circ B-B \circ A,
$$

we can prove that Equation (36) has anti-symmetrical property, and satisfies the Jacobi identical equation, namely, a new product with Lie algebraic structure, then Equation (27) has

Conclusion 2: The snake like-robot systems have Lieadmissible algebraic structure.

\section{THE POISSON'S INTEGRAL METHOD OF SNAKE-LIKE ROBOT SYSTEMS}

The classical Poisson integral method includes setting up the Poisson condition of the first integral for the Hamilton system, the Poisson theorem of third integrals is generated from two known integrals by Poisson brackets. The theoretical foundation of this method includes equations of motion of systems with Lie algebraic structure. Because the snake-like robot system has no Lie algebraic structure, it possesses Lie-admissible algebraic structure. Therefore, part of the Poisson integral methods can be applied to snake-like robot systems. Then we have the following conclusions:

Proposition 1: The necessary and sufficient condition on which $I\left(a^{\mu}, t\right)=c$ is the first integral of snake-like robot systems (27) in that the $I\left(a^{\mu}, t\right)$ satisfies

$$
\frac{\partial I}{\partial t}+[I, H]=0
$$

Proposition 2: $H=c$ is a first integral of snake-like robot systems (27), if the Hamiltonian of the system does not depend explicitly on time $t$, and for $\Lambda_{s}^{\prime}=0$.

Proposition 3: For snake-like robot systems (27), which possess a first integral $I\left(a^{\mu}, t\right)$ that contains $t$, but $H$ and $\Lambda^{\prime}{ }_{s}$ do not depend explicitly on $t$, then

$$
\frac{\partial}{\partial t}\left(\frac{\partial I}{\partial t}\right)+\left[\frac{\partial I}{\partial t} H\right]+\frac{\partial \Lambda_{s}^{\prime}}{\partial t} \frac{\partial I}{\partial a^{\mu}}+\frac{\partial I}{\partial a_{\mu}} S^{\mu \nu} \frac{\partial}{\partial a^{\nu}}\left(\frac{\partial H}{\partial t}\right)=0
$$

i.e.,

$$
\frac{\partial}{\partial t}\left(\frac{\partial I}{\partial t}\right)+\left[\frac{\partial I}{\partial t} H\right]=0
$$

Namely, $\frac{\partial I}{\partial t}$ is the first integral of the snake-like robot system (27), and $\frac{\partial I^{2}}{\partial t^{2}}, \ldots$, are also first integrals of the snake-like robot system.

Proposition 4: For snake-like robot systems (27), which possess a first integral $I\left(a^{\mu}, t\right)$ containing $a^{\rho}$, but $H$ and $\Lambda^{\prime}{ }_{s}$ do not depend explicitly on $a^{\rho}, \frac{\partial I}{\partial a^{\rho}}, \frac{\partial I^{2}}{\partial a^{\rho^{2}}}, \cdots$, are also first integrals of the snake-like robot system.

\section{EXAMPLE}

We set up a simple snake-like robot which only has one joint, and choose $q_{1}=\varphi_{1}, q_{2}=x_{h}, q_{3}=y_{h}, q_{4}=\theta_{h}, q_{5}=\gamma_{1}$ as generalized coordinates.

The relationship between the center of gravity of the first joint $\left(x_{1}, y_{1}\right)$ and the position of the snake head $\left(x_{h}, y_{h}\right)$ can be given by

$$
\begin{aligned}
& x_{1}=q_{2}+2 l c\left(q_{4}\right)+l c\left(q_{1}\right), \\
& y_{1}=q_{3}+2 l s\left(q_{4}\right)+l s\left(q_{1}\right),
\end{aligned}
$$

so, the velocities of snake-like systems can be given by

$$
\begin{aligned}
& \dot{x}_{1}=\dot{q}_{2}-2 l s\left(q_{4}\right) \dot{q}_{4}-l s\left(q_{1}\right) \dot{q}_{1}, \\
& \dot{y}_{1}=\dot{q}_{3}+2 l c\left(q_{4}\right) \dot{q}_{4}+l c\left(q_{1}\right) \dot{q}_{1} .
\end{aligned}
$$

The constraints of the snake-like robot system are given by

$$
\begin{aligned}
f_{\beta}(q, \dot{q})= & \dot{q}_{2} s\left(q_{1}\right)-\dot{q}_{3} c\left(q_{1}\right)-2 l c\left(q_{4}-q_{1}\right) \dot{q}_{4} \\
& -l \dot{q}_{1}-\dot{q}_{5} R=0 .
\end{aligned}
$$

We obtain the kinetic energy of the snake-like robot system

$$
\begin{aligned}
T= & \frac{1}{2}\left(m+m_{h}\right)\left(\dot{q}_{2}^{2}+\dot{q}_{3}^{2}\right)+\frac{1}{2}\left(J_{h}+2 m l^{2}\right) \dot{q}_{4}^{2}+\frac{1}{2}\left(J_{y}+\frac{1}{2} m l^{2}\right) \\
& \dot{q}_{1}^{2}+2 m \dot{q}_{4}\left(\dot{q}_{2} s\left(q_{4}\right)-\dot{q}_{3} c\left(q_{4}\right)\right) \\
& +m l \dot{q}_{1}\left[\dot{q}_{2} s\left(q_{1}\right)-\dot{q}_{3} c\left(q_{1}\right)\right]+2 m l^{2} \dot{q}_{4} \dot{q}_{1}\left(\dot{q}_{4}+\dot{q}_{1}\right) \\
& s\left(q_{4}+q_{1}\right)+\frac{1}{2} J_{r} \dot{q}_{5}^{2},
\end{aligned}
$$

potential energy

$$
U=0 \text {, }
$$

and dissipative function

$$
\begin{aligned}
D= & \frac{1}{2} D_{y} \dot{q}_{1}^{2}+\frac{1}{2} D_{x y}\left[\dot{q}_{2}^{2}+\dot{q}_{3}^{2}+4 l \dot{q}_{4}^{2}+l^{2} \dot{q}_{1}^{2}+4 l \dot{q}_{1}\left(\dot{q}_{2} s\left(q_{4}\right)\right.\right. \\
& \left.-\dot{q}_{3} c\left(q_{4}\right)\right) \\
& \left.+2 l \dot{q}_{1}\left(\dot{q}_{2} s\left(q_{1}\right)-\dot{q}_{3} c\left(q_{1}\right)\right)+4 l^{2} \dot{q}_{4} \dot{q}_{1} s\left(q_{4}+q_{1}\right)\right] .
\end{aligned}
$$

The Lagrange function of the system is written in the form

$$
L=\frac{1}{2}\left(m+m_{h}\right)\left(\dot{q}_{2}^{2}+\dot{q}_{3}^{2}\right)+\frac{1}{2}\left(J_{h}+2 m l^{2}\right) \dot{q}_{4}^{2}+\frac{1}{2}\left(J_{y}+\frac{1}{2} m l^{2}\right)
$$




$$
\begin{aligned}
& \dot{q}_{1}^{2}+2 m l \dot{q}_{4}\left(\dot{q}_{2} s\left(q_{4}\right)-\dot{q}_{3} c\left(q_{4}\right)\right) \\
& +m l \dot{q}_{1}\left[\dot{q}_{2} s\left(q_{1}\right)-\dot{q}_{3} c\left(q_{1}\right)\right]+2 m l^{2} \dot{q}_{4} \dot{q}_{1}\left(\dot{q}_{4}+\dot{q}_{1}\right) \\
& s\left(q_{4}+q_{1}\right)+\frac{1}{2} J_{r} \dot{q}_{5}^{2} .
\end{aligned}
$$

Taking the generalized momenta of snake-like robot systems

$$
\begin{aligned}
p_{1}= & \left(J_{y}+m l^{2}\right) \dot{q}_{1}+m l\left(\dot{q}_{2} s\left(q_{1}\right)-\dot{q}_{3} s\left(q_{1}\right)\right)+2 m l^{2} \dot{q}_{4} \\
& s\left(q_{4}+q_{1}\right), \\
p_{2}= & m_{h} \dot{q}_{2}+2 m l \dot{q}_{4} s\left(q_{4}\right)+m l \dot{q}_{1} s\left(q_{1}\right), \\
p_{3}= & m_{h} \dot{q}_{3}-2 m \dot{q}_{4} s\left(q_{4}\right)-m l \dot{q}_{1} c\left(q_{1}\right), \\
p_{4}= & \left(J_{h}+4 m l^{2}\right) \dot{q}_{4}+2 m l\left(\dot{q}_{2} s\left(q_{4}\right)-\dot{q}_{3} c\left(q_{4}\right)\right)+2 m l^{2} \dot{q}_{1} \\
& s\left(q_{4}+q_{1}\right), \\
p_{5}= & J_{r} \dot{q}_{5},
\end{aligned}
$$

using Equation (47), we can obtain the generalized velocities in the form

$$
\begin{aligned}
& \dot{q}_{1}=\frac{m l s\left(q_{1}\right)-\left(J_{y}+m l^{2}\right) m l c\left(q_{1}\right)}{\left(J_{y}+m l^{2}\right) m l c\left(q_{1}\right)} p_{1}, \\
& \dot{q}_{2}=\frac{2 m l c\left(q_{1}\right)+m l s\left(q_{4}+q_{1}\right)}{m l\left(J_{h}+4 m l^{2} s\left(q_{1}\right)+m l\right)} p_{2}, \\
& \dot{q}_{3}=\frac{\left(J_{y}+m l^{2}\right)\left(J_{y}+m l^{2}\right) m l+2 m l^{2} s\left(q_{4}+q_{1}\right) m l c\left(q_{1}\right)}{4 m l\left(s\left(q_{1}\right)-s\left(q_{1}\right)\right)\left(J_{y}+m l^{2}\right)} p_{3}, \\
& \dot{q}_{4}=\frac{m_{h} m l s\left(q_{4}\right)\left(s\left(q_{1}\right)-s\left(q_{1}\right)\right)}{2 m l} p_{4}, \\
& \dot{q}_{5}=\frac{P_{5}}{J_{r}} .
\end{aligned}
$$

The Hamiltonian of the snake-like robot system can be expressed as

$$
\begin{aligned}
& H=\frac{1}{2} \frac{m l s\left(q_{1}\right)-\left(J_{y}+m l^{2}\right) m l c\left(q_{1}\right)}{\left(J_{y}+m l^{2}\right) m l c\left(q_{1}\right)} p_{1}^{2} \\
& +\frac{m l c\left(q_{1}\right)+m l s\left(q_{4}+q_{1}\right)}{m l\left(J_{h}+4 m l^{2} s\left(q_{1}\right)+m l\right)} p_{2}^{2} \\
& +\frac{\left(J_{y}+m l^{2}\right)\left(J_{y}+m l^{2}\right) m l+2 m l^{2} s\left(q_{4}+q_{1}\right) m l c\left(q_{1}\right)}{4 m l\left(s\left(q_{1}\right)-s\left(q_{1}\right)\right)\left(J_{y}+m l^{2}\right)} p_{3}^{2} \\
& +\frac{m l\left(J_{y}+m l^{2}\right)+\left(s\left(q_{1}\right)-s\left(q_{1}\right)\right)}{J_{h}+4 m l^{2} s\left(q_{1}\right)} p_{4}^{2}+\frac{1}{2} \frac{P_{5}^{2}}{J_{r}} .
\end{aligned}
$$

Using Equations (47)-(49), we can obtain

$$
\begin{aligned}
& \dot{p}_{1}=\frac{1}{2} \frac{m l c\left(q_{1}\right)+\left(J_{y}+m l^{2}\right)}{\left(J_{y}+m l^{2}\right) m l c^{2}\left(q_{1}\right)} p_{1}^{2} \\
& +\frac{m l s\left(q_{1}\right)+m l c\left(q_{4}+q_{1}\right)}{m l\left(J_{h}+4 m l^{2} s^{2}\left(q_{1}\right) c\left(q_{1}\right)+m l\right)} p_{2}^{2}+ \\
& \frac{2 m l^{2} c\left(q_{4}+q_{1}\right) m l c\left(q_{1}\right)}{4 m l\left(c\left(q_{1}\right)-s^{2}\left(q_{1}\right)\right)\left(J_{y}+m l^{2}\right)} p_{3}^{2} \\
& +\frac{m l\left(J_{y}+m l^{2}\right)+\left(c\left(q_{1}\right)+s\left(q_{1}\right)\right)}{J_{h}+4 m l^{2} c\left(q_{1}\right)} p_{4}^{2}
\end{aligned}
$$

$$
\begin{aligned}
& \quad+\frac{1}{2} D_{y} p_{1} m l c\left(q_{1}\right)+D_{x y} p_{3} p_{4} s\left(q_{4}+q_{1}\right)+m l^{2} p_{1} c\left(q_{1}\right) \\
& \dot{p}_{2}=+\frac{m l c\left(q_{1}\right)+m l s\left(q_{4}+q_{1}\right)}{m l\left(J_{h}+4 m l^{2} s\left(q_{1}\right)+m l\right)} p_{2}^{2} \\
& +\frac{m l\left(J_{y}+m l^{2}\right)+\left(s\left(q_{1}\right)-s\left(q_{1}\right)\right)}{4 m l^{2} s\left(q_{1}\right)} p_{4}^{2} \\
& +D_{y} m l p_{5} p_{1} c\left(q_{4}\right)+D_{x y} p_{3}+m l_{2} s\left(q_{1}\right) p_{2}, \\
& \dot{p}_{3}=\frac{m l s\left(q_{1}\right)-\left(J_{y}+m l^{2}\right) m l c\left(q_{1}\right)}{\left(J_{y}+m l^{2}\right) m l c\left(q_{1}\right)} \\
& +\frac{\left(J_{y}+m l^{2}\right)\left(J_{y}+m l^{2}\right) m l+2 m l^{2} s\left(q_{4}+q_{1}\right) m l c\left(q_{1}\right)}{4 m l\left(s\left(q_{1}\right)-s\left(q_{1}\right)\right)\left(J_{y}+m l^{2}\right)} p_{3}^{2} \\
& +D_{y} m l p_{5} p_{1} c\left(q_{4}\right)+D_{x y} p_{3}+J_{h}+4 p_{2} m l^{2} s\left(q_{1}\right)+m l \\
& \dot{p}_{4}=\frac{m l s\left(q_{1}\right)-m l_{c}\left(q_{4}+q_{1}\right)}{m l\left(J_{h}+4 m l^{2} s\left(q_{1}\right)+m l\right)} p_{2}^{2} \\
& +\frac{\left(J_{y}+m l^{2}\right)\left(J_{y}+m l^{2}\right) m l+2 m l^{2} c\left(q_{4}+q_{1}\right) m l c\left(q_{1}\right)}{4 m l\left(s\left(q_{1}\right)-s\left(q_{1}\right)\right)\left(J_{y}+m l^{2}\right)} p_{3}^{2} \\
& +D_{y} m l^{2} p_{4}^{\prime} p_{1} s\left(q_{1}+q_{4}\right)+2 m l D_{x y} p_{2}+J_{y}+m l^{2} \\
& \dot{p}_{5}=J_{r} p_{5}+D_{y} m^{2} l^{2} p_{2} p_{1} c\left(q_{4}\right)+D_{x y} p_{2} p_{4} \\
& +m l p_{2} s\left(q_{1}\right) p_{2},
\end{aligned}
$$

We call Equations (48) and (50) the generalized Hamilton canonical equations of the snake-like robot system.

In which

$$
\left\{\begin{array}{l}
\Lambda_{1}^{\prime}=\frac{1}{2} D_{y} p_{1} m l c\left(q_{1}\right)+D_{x y} p_{3} p_{4} s\left(q_{4}+q_{1}\right)+m l^{2} p_{1} c\left(q_{1}\right), \\
\Lambda_{2}^{\prime}=D_{y} m l p_{5} p_{1} c\left(q_{4}\right)+D_{x y} p_{3}+m l p_{2} s\left(q_{1}\right) p_{2}, \\
\Lambda_{3}^{\prime}=D_{y} m l p_{5} p_{1} c\left(q_{4}\right)+D_{x y} p_{3}+J_{h}+4 p_{2} m l^{2} s\left(q_{1}\right)+m l,(51) \\
\Lambda_{4}^{\prime}=D_{y} m l^{2} p^{\prime}{ }_{4} p_{1} s\left(q_{1}+q_{4}\right)+2 m l D_{x y} p_{2}+J_{y}+m l^{2}, \\
\Lambda_{5}^{\prime}=\mathrm{D}_{y} m^{2} l^{2} p_{2} p_{1} c\left(q_{4}\right)+D_{x y} p_{2} p_{4}+m l p_{2} s\left(q_{1}\right) p_{2} .
\end{array}\right.
$$

Let

$$
\begin{aligned}
& a_{1}=q_{1}, a_{2}=q_{2}, a_{3}=q_{3}, a_{4}=q_{4}, a_{5}=q_{5}, \\
& a_{6}=p_{1}, a_{7}=p_{2}, a_{8}=p_{3}, a_{9}=p_{4}, a_{10}=p_{5},
\end{aligned}
$$

then Equation (50) can be expressed in the contravariant algebraic form

$$
\dot{a}^{\mu}-S^{\mu \nu} \frac{\partial H}{\partial a^{\nu}}=0(\mu \nu=1 \cdots 5,)
$$

where

$$
\begin{aligned}
S^{\mu v} & =\omega^{u v}+T^{u v} \\
\omega^{\mu v} & =\left(\begin{array}{cc}
0_{5 \times 5} & I_{5 \times 5} \\
-I_{5 \times 5} & 0_{5 \times 5}
\end{array}\right), T^{\mu v}=\left(\begin{array}{cc}
0_{5 \times 5} & 0_{5 \times 5} \\
0_{5 \times 5} & -\Omega_{k \times k}
\end{array}\right)
\end{aligned}
$$

using Equations (25) and (51), we have

$$
\left\{\begin{array}{l}
-\Omega_{11}=\frac{1}{2} D_{y} a_{1} m l c\left(a_{1}\right)+D_{x y} p_{3} p_{4} s\left(a_{4}+a_{1}\right)+2 s\left(a_{4}\right) m l^{2} p_{1} c\left(a_{1}\right) \\
-\Omega_{22}=D_{y} m l p_{5} p_{1} c\left(a_{4}\right)+D_{x y} p_{1}+m l a_{4} s\left(a_{1}\right) a_{5} \\
-\Omega_{33}=2 D_{y} m l p_{5} a_{3} c\left(q_{4}\right)+D_{x y} p_{1}+J_{h}+4 a_{4} m l^{2} s\left(a_{1}\right)+m l \\
-\Omega_{44}=D_{y} m l^{2} p_{3} a_{1} s\left(a_{1}+a_{4}\right)+2 m l D_{x y} p_{1}+J_{y}+m l^{2} \\
-\Omega_{55}=D_{y} m^{2} l^{2} p_{3} p_{1} c\left(a_{4}\right)+D_{x y} p_{1} p_{4}+m l a_{7} s\left(a_{1}\right) p_{2} .
\end{array}\right.
$$


Substituting Equations (53) and (54) into Equation (27) leads to the contravariant algebraic form of snake-like robot systems

$$
\left\{\begin{array}{l}
\dot{a}_{1}=\frac{m l s\left(a_{1}\right)-\left(J_{y}+m l^{2}\right) m l c\left(a_{1}\right)}{\left(J_{y}+m l^{2}\right) m l c\left(a_{1}\right)} p_{1}+2 m l^{2} s\left(a_{4}+a_{1}\right) m l c\left(a_{1}\right) p_{2} \\
\dot{a}_{2}=\frac{2 m l c\left(a_{1}\right)+m l s\left(a_{4}+a_{1}\right)}{m l\left(J_{h}+4 m l^{2} s\left(a_{1}\right)+m l\right)} p_{1} \\
\dot{a}_{3}=\frac{\left(J_{y}+m l^{2}\right)\left(J_{y}+m l^{2}\right) m l+2 m l^{2} s\left(a_{4}+a_{1}\right) m l c\left(a_{1}\right)}{4 m l\left(s\left(a_{1}\right)-s\left(a_{1}\right)\right)\left(J_{y}+m l^{2}\right)} p_{3} \\
\dot{a}_{4}=\frac{m h m l\left(a_{4}\right)\left(s\left(a_{1}\right)-c\left(a_{1}\right)\right)}{2 m l} p_{1} \\
\dot{a}_{5}=\frac{p_{1}}{J_{r}}+2 m l^{2} c\left(a_{4}+a_{1}\right) m l c\left(a_{1}\right) \\
\dot{p}_{1}=\frac{1}{2} \frac{m l c\left(a_{1}\right)+\left(J_{y}+m l^{2}\right)}{\left(J_{y}+m l^{2}\right) m l c^{2}\left(a_{1}\right)} p_{1}^{2}+\frac{m l s\left(a_{1}\right)+m l c\left(a_{4}+a_{1}\right)}{m l\left(J_{h}+4 m l^{2} s^{2}\left(a_{1}\right) c\left(a_{1}\right)+m l\right)} p_{2}^{2}+ \\
\frac{2 m l^{2} c\left(a_{4}+a_{1}\right) m l c\left(a_{1}\right)}{4 m l\left(c\left(a_{1}\right)-s^{2}\left(a_{1}\right)\right)\left(J_{y}+m l^{2}\right)} p_{4}^{2}+\frac{1}{2} D_{y} p_{1} m l c\left(a_{1}\right)+D_{x y} p_{4} p_{3} s\left(a_{4}+a_{1}\right) \\
\dot{p}_{2}=\frac{m l c\left(a_{1}\right)+m l s\left(a_{4}+a_{1}\right)}{m l\left(J_{h}+4 m l^{2} s\left(a_{1}\right)+m l\right)} p_{1}^{2}+\frac{m l\left(J_{y}+m l^{2}\right)+\left(s\left(a_{1}\right)-c\left(a_{1}\right)\right)}{4 m l^{2} s\left(a_{1}\right)} p_{4}^{2} \\
+D_{y} m l p_{5} p_{1} c\left(q_{4}\right)+D_{x y} p_{3}+m l p_{2} s\left(a_{1}\right) p_{2} \\
\dot{p}_{3}=\frac{m l s\left(a_{1}\right)-\left(J_{y}+m l^{2}\right) m l c\left(a_{1}\right)}{\left(J_{y}+m l^{2}\right) m l c\left(a_{1}\right)}+D_{y} m l p_{5} p_{1} c\left(a_{4}\right)+D_{x y} p_{3}+J_{h} \\
+4 p_{2} m l^{2} s\left(a_{1}\right) \\
\dot{p}_{4}=\frac{m l s\left(a_{1}\right)-m l c\left(a_{4}+a_{1}\right)}{m l\left(J_{h}+4 m l^{2} s\left(a_{1}\right)+m l\right)} p_{2}^{2}+\frac{\left(J_{y}+m l^{2}\right) m l+2 m l^{2} c\left(a_{4}+a_{1}\right) m l c a_{1}}{4 m l\left(s\left(a_{1}\right)-s\left(a_{1}\right)\right)\left(J_{y}+m l^{2}\right)} p_{4}^{2} \\
\dot{p}_{5}=D_{y} m^{2} l^{2} p_{2} p_{1} c\left(a_{4}\right)+D_{x y} p_{3} p_{4}+m l p_{2} s\left(a_{1}\right) p_{3}
\end{array}\right.
$$

From Proposition (2), the Hamiltonian of the system is written in the form

$$
\begin{aligned}
H= & \frac{1}{2} \frac{m l s\left(a_{1}\right)-\left(J_{y}+m l^{2}\right) m l c\left(a_{1}\right)}{\left(J_{y}+m l^{2}\right) m l c\left(a_{1}\right)} p_{1}{ }^{2} \\
& +\frac{m l c\left(a_{1}\right)+m l s\left(a_{4}+a_{1}\right)}{m l\left(J_{h}+4 m l^{2} s\left(a_{1}\right)+m l\right)} p_{2}{ }^{2}+ \\
& \frac{\left(J_{y}+m l^{2}\right)\left(J_{y}+m l^{2}\right) m l+2 m l^{2} s\left(a_{4}+a_{1}\right) m l c\left(a_{1}\right)}{4 m l\left(s\left(a_{1}\right)-c\left(a_{1}\right)\right)\left(J_{y}+m l^{2}\right)} p_{3}{ }^{2} \\
& +\frac{m l\left(J_{y}+m l^{2}\right)+\left(s\left(a_{1}\right)-c\left(a_{1}\right)\right)}{J_{h}+4 m l^{2} s\left(a_{1}\right)} p_{4}{ }^{2}+\frac{1}{2} \frac{p_{5}{ }^{2}}{J_{r}}=\mathrm{C}_{1},(57)
\end{aligned}
$$

and is the first integral.

Using Proposition (1), we can obtain the following integrals:

$$
\begin{aligned}
I_{1}= & \int \frac{m l s\left(a_{1}\right)-\left(J_{y}+m l^{2}\right) m l c\left(a_{1}\right)}{\left(J_{y}+m l^{2}\right) m l c\left(a_{1}\right)} d t \\
& +4 m l\left(s\left(a_{1}\right)-c\left(a_{1}\right)\right) s\left(a_{4}+a_{1}\right) p_{2}{ }^{2}=\mathrm{C}_{2}, \\
I_{2}= & \int 2 m l^{2} s\left(a_{4}+a_{1}\right) m l c\left(a_{1}\right) p_{1}+D_{y} 4 m l^{2} s\left(a_{1}\right) d t \\
& +\frac{m l^{2} D_{x y} s\left(a_{4}\right) p_{2} a_{5}}{4 m l^{2} s\left(a_{1}\right) c\left(a_{4}+a_{1}\right)}+2 m l s\left(a_{1}\right) a_{5} p_{3}=C_{3},
\end{aligned}
$$

\section{REFERENCES}

1. Chen L, WangYC, Li B. Persent state and future direction towards Snakerobot research. Robot. (2002) 24:559-63. doi: 10.1007/s11769-002-0038-4

2. Hirose S. Biologically Inspired Robots: Snake-Like Locomotors and Manipulators. Oxford: Oxford University Press (1993). p. 351-63.

3. Liljebäck P, Pettersen, KY, Stavdahl $\varnothing$, Gravdahl JT. A review on modelling, implementation, and control of snake robots. Rob Auton Syst. (2012) 60:2940. doi: 10.1016/j.robot.2011.08.010

$$
\begin{aligned}
I_{3}= & \int 2 D_{y} m l^{2} p_{2} s\left(a_{4}+a_{1}\right) m l c\left(a_{1}\right) d t \\
& +m l D_{x y}\left(J_{h}+4 m l^{2} s\left(a_{1}\right)+m l\right)=C_{4} .
\end{aligned}
$$

The first integral $I_{2}$ includes $a_{5}$.Using Proposition (4), from $I_{2}$ we obtain a new integral.

$$
I_{4}=\frac{m l^{2} D_{x y} s\left(a_{4}\right) a_{7}}{4 m l^{2} s\left(a_{1}\right) c\left(a_{4}+a_{1}\right)}+2 m l s\left(a_{1}\right) p_{3}=C_{4} .
$$

\section{CONCLUSION}

In this paper, we have studied the algebraic structure and Poisson integral theory of snake-like robot systems. This method reduces the expression variables of the snake-like robot and makes the expression more concise. We can also obtain the algebraic structure and Poisson integral theory of other soft robots.

\section{DATA AVAILABILITY STATEMENT}

The original contributions presented in the study are included in the article/supplementary material, further inquiries can be directed to the corresponding author/s.

\section{AUTHOR CONTRIBUTIONS}

FJ-L is responsible for the topic selection and research work of the paper. ML is responsible for the research work of the paper. $\mathrm{XC}$ participates in the research of the topic selection and the establishment of motion equation.

\section{FUNDING}

This work was Supported by National Natural Science Foundation of China: 11872335 750000RMB, 11472247 900000RMB.

\section{ACKNOWLEDGMENTS}

The authors of this manuscript acknowledge support from the National Natural Science Foundation of China (Grant Nos. $11872335,11472247)$. 
8. Tang CQ, Ma SG, Li B, Wang MH, Wang WC. The study of snake robots biomimetic control method with the environments adaptability. Chin Sci Inform Sci. (2014) 44:647-63. doi: 10.1360/112011-1341

9. Liljeback P, Pettersen KY, Stavdahl O, Gravdahl JT. Compliant control of the body shape of snake robots. IEEE Int Conf Rob Autom. (2014) 31:454855. doi: 10.1109/ICRA.2014.6907523

10. Liljeback P, Pettersen KY, Stavdahl O. Modelling and control of obstacle-aided snake robot locomotion based on jam resolution. IEEE Int Conf Rob Autom. (2009) 15:3807-14. doi: 10.1109/ROBOT.2009.5152273

11. Ostrowski J, Burdick J. Gait kinematics for a serpentine robot. IEEE Int Conf Rob Autom. (1994) 2:1294-9. doi: 10.1109/ROBOT.1996.506885

12. Guo X, Ma SG, Li BM, Wang H, Wang YC. Modeling and optimal torque control of a snake-like robot based on the fiber bundle theory. Sci China. (2015) 58:183-95. doi: 10.1007/s11432-014-5253-y

13. Mei FX. Applications of Lie Groups and Lie Algebras to Constrained Mechanical Systems. Beijing: Science Press (1999). p. 1-167.

14. Mei FX. On the integration methods of non-holonomic dynamics. Int J Non Linear Mech. (2000) 35:229-38. doi: 10.1016/S0020-7462(99)00011-6

15. Mei FX, Shi RC. The algebraic structure and the poisson integral method of the nonholonomic systems. J Beijing Inst Technol. (1996) 13:51-5.
16. Fu JL. The algebraic structure and poisson integral procedure of dynamic equation for relativistic Birkhoof's systems. Acta Math Sci. (2001) 21:70-8.

17. Fu JL, Chen XW, Luo SK. Algebraic structure and poisson integrals of relativistic dynamical equations for rotational systems. Appl Math Mech. (1999) 20:1266-74. doi: 10.1007/BF02463795

18. Fu JL, Xie FP, Guo YX. Algebraic structure and Poisson's integral theoryof $f(R)$, Cosmology. Int. J.Theor. Phys. (2012) 51:35-48. doi: 10.1007/s10773-011-0875-y

Conflict of Interest: The authors declare that the research was conducted in the absence of any commercial or financial relationships that could be construed as a potential conflict of interest.

Copyright (c) 2021 Jing-Li, Chun and Lei. This is an open-access article distributed under the terms of the Creative Commons Attribution License (CC BY). The use, distribution or reproduction in other forums is permitted, provided the original author(s) and the copyright owner(s) are credited and that the original publication in this journal is cited, in accordance with accepted academic practice. No use, distribution or reproduction is permitted which does not comply with these terms. 